\title{
Del riesgo a la resiliencia. Hacia el diseño y gestión adaptativo
}

del paisaje

\section{From risk to resilience. Towards the design and adaptive management of}

the landscape

\author{
Miriam Garcia Garcia* \\ Resident Professor, Master Degree Landscape Architecture, Politécnico de Milán (IT) \\ Profesora. Máster en Arquitectura de Paisaje, Universidad Politécnica de Catalunya (ES) \\ Profesora. Máster Universitario en Urbanismo y Estudios Territoriales, Instituto Nacional de Adminis- \\ tración Pública en Madrid (ES) \\ Profesora Visitante. Maestría en diseño del Paisaje, Universidad Pontificia Bolivariana (CO) \\ miriamgarcia@landlab.es
}




\section{Resumen}

Habitamos en una época denominada como 'Antropoceno' donde el calentamiento global, comúnmente conocido como cambio climático, es al mismo tiempo su conición y su crisis más emblemática. Una época donde la falta de control y desconocimiento de las consecuencias de las decisiones de la sociedad en relación al medio han hecho surgir una renovada conciencia global del riesgo. Esta reflexión global debe tener su traslación en una renovación del campo del diseño y la planificación. Una práctica que se aleje de la idea de control del riesgo y que explícitamente dote de capacidad de resiliencia a los sistemas, posibilitando de este modo su adaptación a largo plazo y propiciando su viabilidad ecológica, cultural y económica. Trabajar desde la resiliencia permite entonces unir ecología y planificación a través de la gestión adaptativa mediante el diseño y la experimentación.

Palabras claves: Cambo climático; Riesgo; Resiliencia; Paisaje

\section{Abstract}

We live in a time called, Anthropocene' where global warming, commonly known as climate change, is at the same time its condition and its most emblematic crisis. An era where the lack of control and knowledge of the consequences of the decisions of society regarding the environment have given rise to a renewed global awareness of risk. This global reflection must promote a renewal in the field of design and planning. A practice that moves away from the idea of risk control and that explicitly endows the systems with resilience, enabling their long-term adaptation and fostering their ecological, cultural and economic viability. Working with resilience then makes it possible to merge ecology and planning through adaptive management through design and experimentation.

Keywords: Climate change; Risk; Resilience; Landscape 


\section{La función alumbradora del riesgo}

El concepto de riesgo es una de las principales herramientas teóricas de las que dispone la sociología para dar cuenta de las dinámicas propias de la sociedad contemporánea. No sabemos si vivimos en un mundo con más riesgos que los de anteriores civilizaciones. Lo cierto, es que la sociedad actual ha sido definida por algunos sociólogos como Ulrich Beck y Niklas Luhmann como una sociedad en riesgo, para referirse a que vivimos en un mundo fuera de control, donde nada es seguro menos la incertidumbre. No se trata por lo tanto de la cantidad de riesgo, como de la falta de control y desconocimiento de las consecuencias de las decisiones de la sociedad en relación al medio, lo que diferencia este momento de la historia (Beck, 1999).

Lo contradictorio de este momento es que a pesar de este contexto de incertidumbre el concepto de riesgo sigue estando vinculado, especialmente en campo del diseño y de la planificación (pero también en otros como la economía o la política), a un mundo gobernado exclusivamente por las leyes de la probabilidad, en el que todo es medible y calculable. Por ello, no es extraño que los proyectos llevados a cabo en base a esta noción de riesgo hayan amplificado en numerosas ocasiones los riesgos que querían evitarse. Al menos una década antes de la devastación del huracán Katrina, ecologistas e hidrólogos ya habían advertido que el enfoque del control de inundaciones del Cuerpo de Ingenieros del Ejército de los Estados Unidos (USACE) estaba empujando a la cuenca baja del Mississippi hacia un umbral catastrófico y el posible colapso. La política llevada a cabo de protección contra las inundaciones mediante diques y represas, junto con la eliminación de humedales costeros y la ocupación y explotación intensiva de las planicies aluviales, hicieron que los mecanismos naturales de adaptación a las inundaciones de la cuenca deltaica se vieran afectados. Cuando la gran tormenta del huracán Katrina azotó el área en 2005, los peores pronósticos se cumplieron. Hizo falta algo más que la evidencia científica, una gran catástrofe, para redefinir la gestión del paisaje en el área.
Figuras 1, 2 y 3: Portadas revista TIME septiembre 2005 y balance sedimentario (1935-2010).
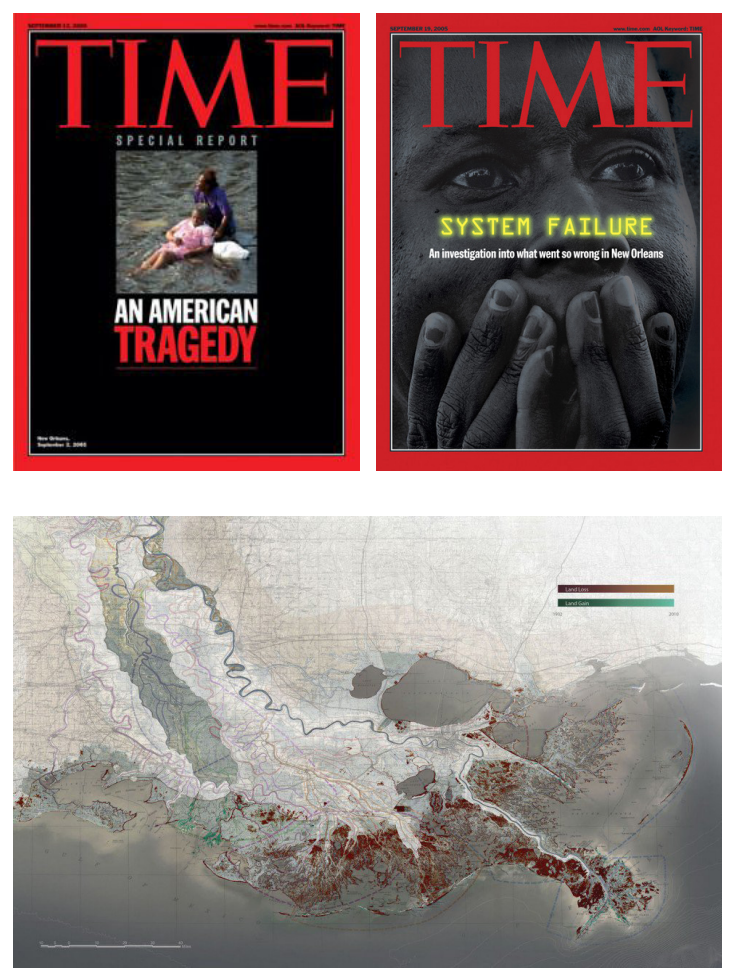

Fuente: LSU Coastal Sustainability Studio.

Esta noción de riesgo con la que se ha venido planificando y diseñando a ambos lados del Atlántico se ha demostrado falible y las catástrofes ambientales, entre otras, lo han puesto en evidencia. The Global Risks Report 2019,1 , último informe elaborado por el Foro Económico Mundial, refleja que los riesgos ambientales, representan tres de los cinco riesgos principales por probabilidad y cuatro por impacto, dominando la encuesta anual de percepción de riesgos globales por tercer año consecutivo.

¿Es posible entonces encontrar una relación entre el sentimiento de riesgo global que domina nuestro tiempo y la creación de una nueva conciencia? Y si es así, qué implicaciones tiene para la planificación y el diseño esta nueva conciencia del riesgo. Para explicar esta relación Ulrich Beck recurre al filósofo americano John

1. Global Risks Report 2019,14a edición, es publicado por el Foro Económico Mundial. 
Dewey quien en su libro publicado en 1927 The Public and its Problems, argumenta que no son las acciones, sino sus consecuencias, las que conforman el corazón de la política y de una nueva conciencia. Así, para Dewey, la actividad científica consiste más que en buscar un fundamento último de la realidad, en ir desarrollando progresivamente hábitos que respondan más eficazmente a los problemas del hombre en la Tierra. Es en esta interacción social de los seres humanos con el entorno, donde surgen órdenes y formas de conducta comunitaria que permiten la adaptación progresiva al mismo (Dewey 1929).

Claramente Dewey no estaba pensando entonces en el cambio climático, pero sin duda su discurso postmoderno es aplicable a este campo en nuestros días. Se puede decir entonces que existe otra noción de riesgo, aquella utilizada para referirse a las incertidumbres no cuantitativas, es decir a los riesgos que no pueden ser conocidos. Este es el caso del cambio climático, que responde a lo que el filósofo Timothy Morton define como hiper-objeto. Morton utiliza este concepto para describir objetos que masivamente se distribuyen en tiempo y espacio trascendiendo la especificidad espacio-temporal. Objetos que no podemos comprender y que sólo reconocemos por sus efectos, que son nuevos y característicos de la época actual (Morton 2013).

Y es en este contexto donde Beck, en vez de centrase en las secuelas negativas de los efectos, nos propone reflexionar sobre las consecuencias imprevistas pero positivas y emancipadoras de las catástrofes o los acontecimientos climáticos extremos (Beck 2015). Es lo que denomina como la función esclarecedora o iluminadora, del riesgo. Por que son los momentos en los que las estructuras y órdenes establecidos fracasan los que permiten el alumbramiento de nuevos modelos de auto organización, como gérmenes de nuevos órdenes y tipologías.

Habitamos en una época sin precedentes, llena de incertidumbres no solo en el dominio ambiental, sino también en el social y económico. Esta época ha sido denominada como
'Antropoceno'2 y el calentamiento global, comúnmente conocido como cambio climático, es al mismo tiempo su condición y su crisis más emblemática. Y es que los humanos hemos convertido el mundo progresivamente en un sistema planificado y diseñado, en especial desde el siglo XVIII y la Revolución industrial. $Y$ además lo hemos hecho concibiendo este como un sistema cerrado. Sin embargo, la atmósfera, los océanos y la tierra son sistemas abiertos que, con sus fuerzas biológicas, químicas y físicas han conseguido hasta la fecha aparentemente absorber, en gran medida, los cambios globales. Pero hace tiempo que sabemos que esto ya no es así. Por supuesto, en este escenario como siempre, las dinámicas físicas, biológicas y químicas siguen sus ciclos, pero fuertemente condicionados por la acción humana.

En este contexto, no solo Beck, sino autores como el politólogo Colin Hay o el economista Joseph Schumpeter, reclaman la atención sobre las crisis y catástrofes como momentos de destrucción creativa. Suponen oportunidades históricas para nuevas formas políticas y auto organizativas de intervención, surgidas de la innovación (Hay 1996; Schumpeter 1942).

El reto entonces consiste en trasladar esta reflexión global al campo del diseño y la planificación.

\section{El marco de la resiliencia}

En nuestros días la crisis climática se manifiesta con diferentes intensidades y escalas relacionando la dimensión económica, ecológica, política y social de los territorios y sistemas. En este contexto, el concepto de resiliencia se manifiesta como una herramienta útil para la comprensión de sus relaciones y dinámicas.

Aunque la literatura sobre resiliencia es abundante, sin embargo, es todavía un concepto

2. Paul J. Crutzen y Eugene F. Stoermer propusieron en 2000 un nuevo tiempo geológico, el 'Antropoceno' como consecuencia de la influencia global de las actividades humanas sobre el Sistema Tierra y sus manifestaciones geológicas (Crutzen, Stoermer 2000) 
en construcción, especialmente en lo que a su aplicación se refiere. Se podría decir que básicamente y pesar de sus distintas interpretaciones en las diferentes disciplinas, es posible diferenciar al menos tres variaciones, de complejidad creciente, del concepto de resiliencia: lo que se conoce como resiliencia ingenieril, la resiliencia de sistemas y la resiliencia ecológica (en sistemas adaptativos complejos). La resiliencia ingenieril se utiliza, por ejemplo, en el desarrollo de programas educativos, la resiliencia de sistemas tiene más difusión en el campo de la gobernanza y la gestión y la resiliencia de los sistemas adaptativos complejos se ha utilizado en economía, innovación y tecnología, la historia y la planificación ambiental y del paisaje.

La resiliencia ingenieril implica la capacidad de recuperarse más rápido después del estrés y ser capaz de soportar cada vez un nivel mayor de estrés. En este sentido, ser resiliente tiene que ver con soportar una gran perturbación sin cambiar, desintegrarse, o ser dañado de manera permanente; regresando a la normalidad rápidamente y con la menor distorsión. Esta es probablemente la acepción del concepto más extendida, sin embargo, es también la de menor alcance. Su nombre proviene de sistemas ingenieriles, como puentes u otras infraestructuras, que están diseñadas para soportar grandes situaciones de estrés y recuperarse de manera rápida cuando éstas cesan. También se utiliza en el campo de la psicología aplicado a los seres humanos tras acontecimientos traumáticos para el individuo. Pone el acento tan solo en la disminución del riesgo y la recuperación de las condiciones de partida. Defiende que existe solamente un estado de equilibrio, que los elementos vuelven a ese estado de equilibrio después de una perturbación y que las perturbaciones son esperadas y conocidas. Por lo tanto, se trata de una aproximación que no es aplicable a los ecosistemas, en constante proceso de transformación fruto de características internas y externas a los que están vinculados de manera directa o indirecta. Es importante esta precisión puesto que en base a esta interpretación reduccionista del concepto de resiliencia en el marco de la planificación y del diseño se han llevado a cabo (todavía sigue siendo la mirada más extendida) las grandes estrategias de gestión hidráulica o defensa costera, entre otras.

Una aproximación un poco más amplia es la conocida como resiliencia de sistemas, que al contrario de la Ingenieril basada en la resistencia, lleva implícita el concepto de cambio. De este modo, para que un sistema siga funcionado tras una situación de estrés o de perturbación, el sistema o una parte del mismo, experimenta un cambio. Estos cambios pueden ser tanto del conjunto de elementos del sistema, como de las relaciones entre ellos.

Si bien esta perspectiva es parte fundamental de la Teoría de la evolución defendida por Wallance y luego Darwin, ha ido evolucionando hasta nuestros días traspasando la frontera determinista de la evolución para abrazar la de la capacidad inherente de adaptación regenerativa de los denominados como sistemas adaptativos complejos. Un sistema adaptativo complejo, denominación acuñada en el interdisciplinario Santa Fe Institute por John H. Holland, Murray Gell-Mann y otros, es un tipo de sistema complejo que además de ser diverso y de estar formado por distintos elementos interrelacionados, tiene la capacidad de cambiar y aprender de la experiencia. La biósfera, las comunidades y las ciudades lo son. La tercera acepción del concepto de resiliencia tiene que ver precisamente con esta singularidad de los sistemas adaptativos complejos, con su capacidad de adaptabilidad, que no es solo adaptación o cambio, sino que conlleva la capacidad de generar nuevos modos de auto-organización, fundamentados en el aprendizaje, tras las perturbaciones. Una capacidad que está directamente relacionada con la innovación ante situaciones imprevisibles. Una cualidad imprescindible de incorporar a la planificación y el diseño ante los inciertos efectos del cambio climático.

Sin embargo, la teoría dominante en el siglo XX estaba construida bien sobre la acepción ingenieril del concepto de resiliencia o bien sobre la idea de la sucesión ecológica de las comunidades en la búsqueda de la estabilidad dentro de diferentes medios (Clements, 1916). Frente a estas aproximaciones, en los años 70 del siglo pasado surge el concepto de resiliencia 
ecológica de la mano del ecologista canadiense C. S. Holling (Holling 1973). Defiende que los ecosistemas no evolucionan hacia un único estado de equilibrio, sino a través de periódicos ciclos de cambio. De este modo la resiliencia representa la capacidad de un sistema de absorber y aprender de las perturbaciones a las que se ve sometido consiguiendo salir adelante sin cambios sustanciales en su estructura y función (sin cambiar de estado). Desde este punto de vista un sistema puede ser muy resiliente y fluctuar mucho, es decir tener poca estabilidad. De hecho, según el propio Holling, los sistemas complejos fluctúan más que los sencillos.

Esta acepción del concepto de resiliencia se revela, entonces, como contrapunto al de vulnerabilidad, cuestión que le ha valido su aceptación e integración en otras disciplinas. Tiene por lo tanto un alcance físico, social, económico y medioambiental. Una gestión o un diseño basado en este concepto de resiliencia, enfatiza la necesidad de dejar opciones abiertas, dotar de heterogeneidad al sistema, persistencia, cambio e imprevisibilidad (Holling 1973; 1996).

En el marco de la resiliencia ecológica hay otros dos conceptos interesantes de diferenciar. La denominada resiliencia específica, con la que se denomina a la capacidad de un sistema a responder a una perturbación conocida. Y la resiliencia general, referida a la capacidad de todo el sistema de sobreponerse a diferentes perturbaciones o cambios. Esta distinción es importante, porque si nos centramos en la resiliencia específica, el sistema en su conjunto se puede volver menos diverso, menos flexible, menos receptivo a interrelaciones y, por lo tanto, más vulnerable (Walker and Salt. 2006). Es aquí cuando el concepto de la escala, propio del campo de la planificación, cobra sentido como complementario al del diseño, ya que esta característica supone analizar los eventos desde una perspectiva regional en vez de local. O mejor expresado, implica una aproximación multiescalar. Es decir, un continuo subir y bajar de escala, en relación a los elementos del sistema en cada uno de los distintos ciclos adaptativos. De este modo, pequeñas interacciones a pequeña escala se transforman en patrones emergentes a gran escala. Para posibilitar esta capacidad de auto-organización los sistemas han de ser heterogéneos, carecer de linealidad cronológica, contar con una organización jerárquica y flujos.

Desde Holling las definiciones del concepto de resiliencia no han variado mucho, pero si se han ido refinando. Así, para Folke la resiliencia de los sistemas socio ecológicos depende de la capacidad del sistema de absorber las perturbaciones permaneciendo en el mismo estado, alterando su grado de auto-organización y mejorando su adaptación y aprendizaje (Folke 2006). Estableciendo una analogía geométrica se podría decir que los sistemas operan como una serie de capas anidadas e interconectadas que se relacionan e influencian unas con otras. Es precisamente el entendimiento de la estructura y funcionamiento de estos sistemas, sus elementos e interrelaciones, su alcance y escala, lo que permitirá intervenir en los mismos a través del diseño favoreciendo su resiliencia; bien sea a través de la manipulación de su estructura y función, introduciendo repeticiones y/o espacio para que el sistema cambie, o mejorando los mecanismos de retroalimentación y de respuesta adaptativa (Walker at al 2004).

De aquí surgen otros dos conceptos importantes como parte de la resiliencia: la adaptabilidad, o capacidad adaptiva y la capacidad de transformación (Walker et al., 2004; Olsson et al., 2004; Folke et al., 2010). La adaptabilidad, definida como la capacidad de un sistema de mantenerse en su estado de equilibrio (Berkes et al., 2003). De modo que la resiliencia tendría que ver con el mantenimiento de determinados procesos a pesar de los cambios exógenos y endógenos (Carpenter and Brock, 2008). Si además, el sistema es capaz de transformarse parcialmente cuando las estructuras ecológicas, económicas o sociales son insostenibles, nos encontramos más próximos a su adaptabilidad (Walker et al., 2004).

\section{El Ciclo Adaptativo}

Los sistemas socio ecológicos experimentan lo que se conoce como ciclos de cambio adaptativos que operan a diferentes escalas 
espacio temporales (panarquía). Esta es una metáfora que sirve para describir cómo se organiza el sistema en sí mismo y cómo responde a un mundo cambiante. No olvidemos que el cambio climático supone constantes y continuados cambios que se experimentan tanto en la evolución de sus variables lentas (tales como el aumento de la temperatura o subida gradual del nivel del mar) como las variables rápidas (tales como temporales, periodos de intensas lluvias o de sequías prolongadas). Estos ciclos de cambio proceden a través de cuatro fases (o régimenes): crecimiento rápido (explotación), conservación, liberación y reorganización (Gunderson and Holling 2002). Es importante para evaluar la resiliencia conocer la forma en la que el sistema se comporta, ya que difiere de una fase a otra en la fortaleza de las conexiones internas, su potencial y resiliencia.

La primera fase ( $r$ ) es interpretada como de crecimiento rápido; se caracteriza por la disponibilidad de recursos, pero sus componentes están débilmente interconectados y regulados, son muy flexibles y poco vulnerables. Se suelen dar en momentos de fuertes variaciones del ambiente y se desarrollan rápidamente. En los ecosistemas son las malas hierbas o las especies pioneras, mientras que en los sistemas económicos son los emprendedores o startups y productores de nuevos artículos. Son momentos de inestabilidad, pero alta resiliencia. Mientras la estructura y las conexiones entre los componentes del sistema se incrementan, la cantidad de energía requerida para mantenerlo crece.

La progresión en el ciclo va de la fase de crecimiento lentamente hacia la segunda fase (K), de conservación. Es aquella donde el ritmo de crecimiento de la red se ralentiza y el sistema se vuelve más interconectado, más eficiente en el uso de los recursos, suele operar durante periodos más prolongados de tiempo y a mayor escala. Son los momentos en los que se suelen eliminar en favor de la eficiencia del sistema valores como la redundancia (maneras diferentes de realizar la misma función). Cuanto más rígida es su organización, menos flexible y más vulnerable se vuelve a las perturbaciones externas. Es decir, son sistemas muy estables,
Figura 4: Fases de los ciclos adaptativos

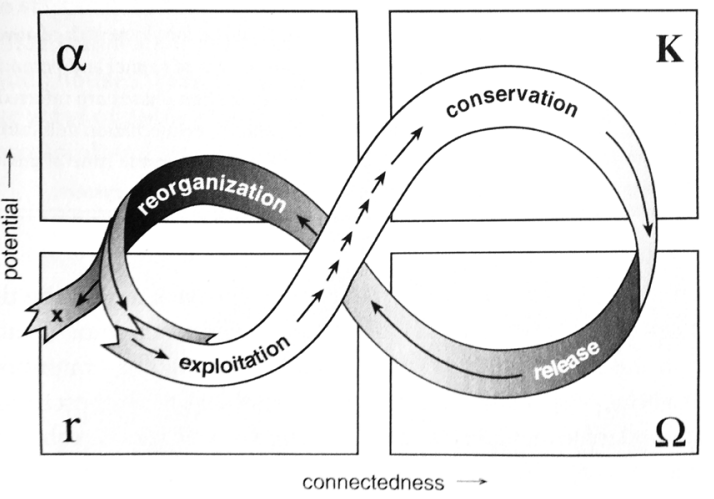

Fuente: Holling y Gunderson (2002). ( las flechas cortas indican cambios lentos y las flechas largas cambios rápidos).

pero solo bajo determinadas condiciones, si esas condiciones cambian bruscamente han perdido su capacidad de auto-organización. Estas dos fases, $r-K$, se integran en un bucle de crecimiento denominado como front loop y tal y como se ha indicado, se corresponden con el proceso de sucesión ecológica de los ecosistemas y constituye los modos de desarrollo en las organizaciones y sociedades.

El paso de la fase k de conservación a la fase conocida como $(\Omega)$, corresponde a la de liberación, y puede suceder en apenas un latido. A veces una pequeña perturbación es suficiente. En realidad, generalmente cuanto más prolongada haya sido la fase de conservación menor ha de ser el shock para pasar a esa fase. En los ecosistemas un fuego, una plaga o una sequía ocasionan la liberación de la biomasa y nutrientes acumulados. Se corresponde también con momentos de shock de mercados. Son periodos caóticos en los que se libera capital que se convertirá en fuente de reorganización y renovación de la siguiente fase. Se corresponde con la fase ya mencionada que el economista Schumpeter denomina como de destrucción creativa. En esos momentos de caos una oportunidad promueve el paso a la fase cuarta ó $(\alpha)$ conocida como de reorganización. Estas dos fases constituyen un segundo bucle llamado back loop. En los ecosistemas pueden ser especies pioneras, semillas resistentes a la perturbación o incluso especies ajenas al lugar y oportunistas las que generen combinaciones 
Figura 5: El ciclo adaptativo (Holling 2001, Gunderson y Holling 2002): una metáfora útil para comprender la innovación radical y progresiva en SES complejos.

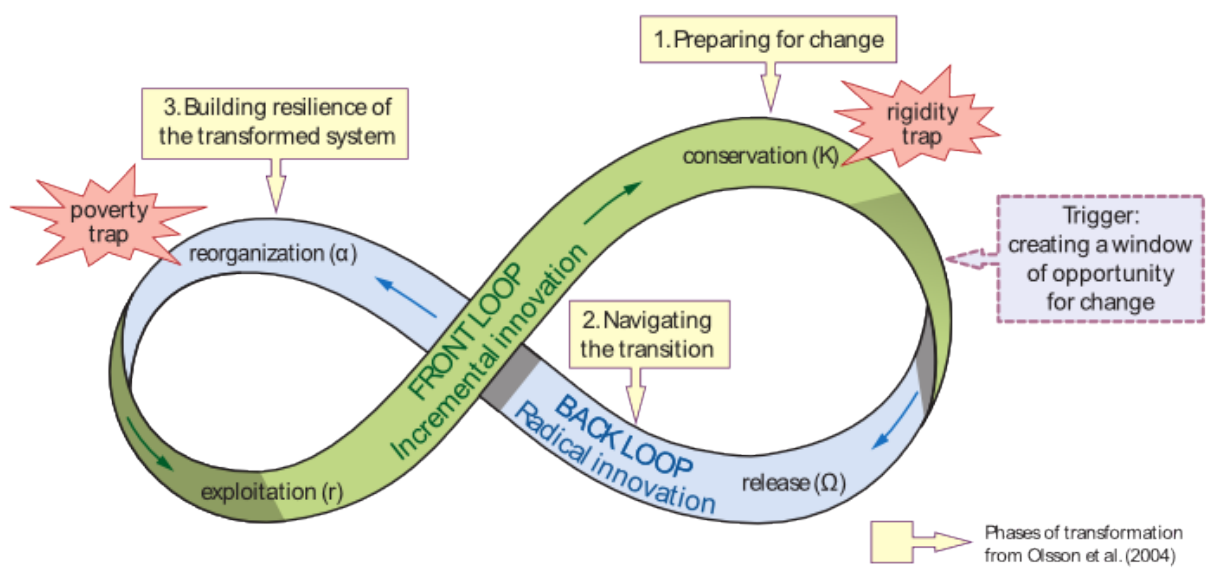

Fuente: Adaptado de Panarchy por Lance H. Gunderson y C. S. Holling, editores.

que posibiliten el crecimiento. En los sistemas sociales y económicos tiene que ver con nuevas organizaciones o empresas creadas a partir de las habilidades y experiencia de organizaciones pasadas. Esta fase suele ser una fase rápida que da paso a una nueva fase de crecimiento tan pronto como se consigue una nueva identidad.

Se puede apreciar una baja resiliencia en la fase de liberación o destrucción creativa, aunque ya con indicios de crecimiento, una conectividad que disminuye reforzando la capacidad de adaptación y el crecimiento del potencial que aumenta las posibilidades de transformación, dando lugar al segundo bucle (back loop) conocido como de desarrollo, reorganización e innovación. En las fases de liberación y reorganización es cuando el sistema socio-ecológico se vuelve más vulnerable al cambio, ya que es precisamente cuando los efectos de los vínculos o las interacciones entre el sistema socio-ecológico y otros sistemas en otras escalas llegan a ser más pronunciados.

Mientras el primer bucle (front loop) es predecible y tiene como objetivo maximizar la producción y acumulación (son momentos de fuerte crecimiento económico y riqueza), el segundo bucle de reorganización (cuyo objetivo es maximizar la innovación y la experimentación), suele ser altamente impredecible y estar lleno de incertidumbre. Esto significa que el ciclo adap- tativo involucra el crecimiento y la estabilidad, por un lado y el cambio y la reorganización por el otro. Pero para que ese cambio se produzca es necesario tener en cuenta que la pérdida o disminución de capital (social, humano, financiero, físico o ambiental, así como por el sistema de instituciones y gobernanza) durante la fase de liberación disminuye las opciones de reorganización para transitar hacia una nueva fase de crecimiento. Por eso, en ocasiones los sistemas colapsan y desaparecen, otras veces entran en estados de degradación (esto es lo que se conoce en los sistemas sociales como trampas de pobreza) y en otras ocasiones, el sistema puede volver a repetir el ciclo anterior o iniciar un ciclo nuevo dotado de una renovada identidad.

Figura 6: Variantes del ciclo adaptativo

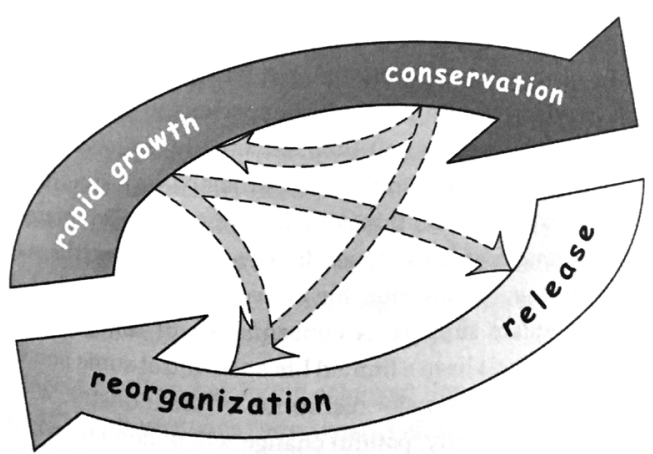

Fuente: Walter and Salt 2006. 
Tal y como muestra la imagen las transiciones entre las fases también son posibles, excepto entre la fase de liberación y reorganización directamente a la fase de conservación. Este aprendizaje es válido para compatibilizar fases de conservación con fases de reorganización y rápido crecimiento y permitir de este modo mantener modelos probados, con sistemas innovadores que avanzan cambios y flexibilizan el sistema o lo preparan para posibles perturbaciones, dotándolo de una mayor resiliencia.

Esta imagen nos muestra cómo los niveles pequeños y rápidos inventan y experimentan, suponen algo así como una pequeña revolución (revolt), mientras que los niveles mayores y lentos estabilizan y conservan la memoria acumulada de sucesos pasados (remember). Es decir, que la interacción entre los ciclos en una panarquía combina creatividad, experimentación y aprendizaje, con continuidad (mantenimiento de las oportunidades y condiciones aprendidas). Estos conceptos se han utilizado para analizar los ciclos o procesos de cambio como oposición a la evolución determinista o al análisis predictivo. Por ejemplo, ha sido utilizado para analizar los cambios en la agricultura (Allison and Hobbs 2004; Evans 2008), o en los procesos participativos (Westley 2008). La idea además es que los niveles rápidos generan agitación y los niveles lentos estabilizan a todo el sistema y lo proveen de memoria e innovación (Gunderson and Holling, 2002). En un bosque, por ejemplo, existen diferentes grupos de especies, cada una de las cuales evoluciona y crece de una forma y con un tiempo distinto. Cuando un fuego, una tormenta o una enfermedad o cualquier otra perturbación les sobreviene causando la muerte de determinadas comunidades, transforma el paisaje. No obstante, algunas especies pioneras son capaces de abrirse paso, desarrollarse y crecer. El paisaje por lo tanto cambia, pero el bosque como tal permanece. Por lo tanto, los sistemas socio ecológicos tienen múltiples posibles estados operacionales y son capaces de pasar de uno a otro. Todos estos estados, al menos desde el punto de vista ecológico, son tan saludables y apropiados como lo demás. No hay un único estado posible para un determinado paisaje. Esta mutabilidad ofrece al mismo tiempo
Figura 7: Panarquía, término utilizado para describir el carácter dinámico y multiescalar de los sistemas socioecológicos

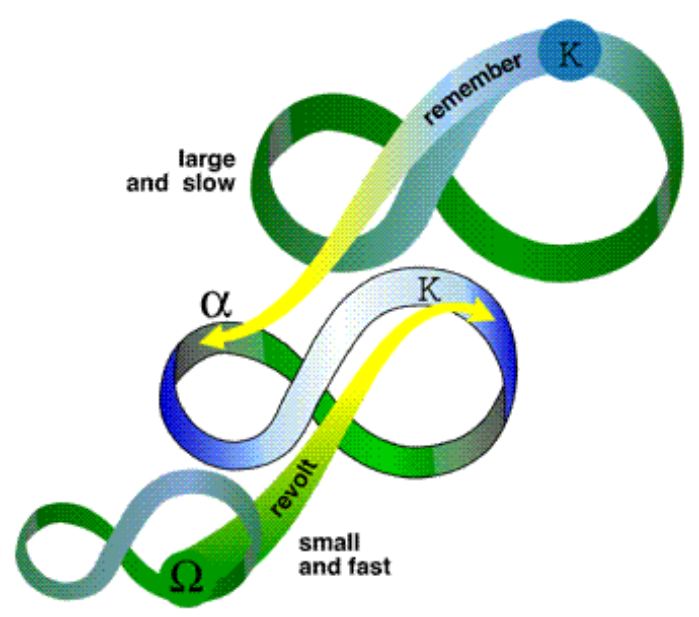

Fuente: Holling, Gunderson y Peterson (2002).

retos y oportunidades para los diseñadores a la hora de definir futuros escenarios posibles de los paisajes. Significa también centrarse en los cambios que experimentan estos sistemas, identificar en qué fase del ciclo adaptativo se encuentran y promover, en consecuencia, acciones concretas de trasformación. Por ejemplo, si la subida del nivel del mar o un fuerte temporal impide el paso de unos campos de cultivo a un humedal, será muy costoso mantener su protección frente a las inundaciones, gestionar su drenaje y las características de salinidad del suelo, además de perder la posibilidad para el conjunto del sistema de contar con la barrera natural de protección frente a la energía de las olas y las inundaciones que suponen estos espacios húmedos e intermareales.

\section{Diseñar la resiliencia ante los efectos del cambio climático}

El cambio climático puede ser considerado un impulsor de cambios regenerativos adaptativos. Ante esto el sistema socio ecológico debe activar sus estrategias de reorganización para recuperar su estado de "estabilidad". La activación de esas estrategias de reorganización es lo que podríamos denominar como construir o incrementar la resiliencia del sistema. Es decir, se trata de activar la capacidad adaptativa del sistema para que este no sobrepase los límites 
que pueden ocasionar que el sistema se precipite a un nuevo estado, iniciando un nuevo ciclo panárquico.

Del conocimiento de los límites del sistema y de las fuerzas ambientales económicas y sociales que los dirigen, así como del funcionamiento de sus ciclos adaptativos, es posible gestionar el sistema desde las bases del pensamiento resiliente. La diversidad, versatilidad ecológica, modularidad, el reconocimiento de las variables lentas, el aprendizaje y rápido retorno, el capital social, la innovación, los solapamientos de gobernanza, así como la valoración de los servicios ecosistémicos, deben guiar los proyectos de resiliencia ante los efectos del cambio climático (Walker and Salt. 2006). Estos atributos definidos por Walker and Salt, a pesar de ser aún muy generales, son útiles porque tienen implicaciones temporales y espaciales, permitiendo su aplicación al campo de la planificación y el diseño a múltiples escalas.

\section{El paisaje como infraestructura resiliente}

Tal y como se ha argumentado, durante décadas, la biología y la ecología han enseñado, y en algunos lugares todavía enseñan, que los ecosistemas evolucionan en estados estables de clímax desde los que no se mueven a menos que se los perturbe. Ejemplos clásicos son la idea de que el bosque madura y luego permanece en ese estado de manera permanente, de modo que cualquier perturbación de ese estado se considere una aberración. Sin embargo, ahora sabemos que el cambio no solo está integrado en estos sistemas, sino que, su capacidad de adaptación depende realmente del él. Todos los ecosistemas están en constante evolución y, a menudo, de manera discontinua y desigual. Si bien percibimos que algunos estados de los ecosistemas son estables, esto no es una estabilidad estricta en un sentido matemático. Se trata tan solo de que nuestra percepción está limitada por el tiempo.

Si la ecología aportó la perspectiva del no equilibrio, la ecología del paisaje define los paisajes como entidades espaciales heterogéneas, con regímenes de perturbación inherentes en términos de tipo, frecuencia e intensidad de la perturbación. Con el foco puesto en las relaciones de los procesos de los patrones del paisaje, la ecología del paisaje ha llevado de manera explícita y sistemática la perspectiva del no equilibrio a la planificación del paisaje (Turner 1989). Hoy en día, la ecología del paisaje abraza también el concepto de gestión adaptativa. Efectivamente, aunque no podremos aspirar a una total gestión de los sistemas vivos, sin embargo, si podemos centrar nuestras energías en proporcionar las condiciones o el contexto que favorezcan su auto organización. Y para ello, es necesario tener en cuenta a todos los actores (humanos y no humanos) del sistema, sus flujos e interrelaciones. Abrazar la resiliencia posibilita entonces unir ecología y planificación a través del diseño de su gestión adaptativa. Esto necesariamente implica una posición proactiva y creativa, experimental y no proteccionista o reactiva, del paisaje.

Si bien es cierto que no se puede reducir el universo del diseño del paisaje a su mera funcionalidad ecológica, debiéndose por lo tanto integrar las cuestiones históricas, culturales, fenomenológicas, identitarias o productivas, no es menos cierto esta dimensión cobra especial importancia de la mano de la compresión de su funcionamiento metabólico y capacidad de resiliencia. Los campos de la ecología del paisaje, el urbanismo ecológico y más recientemente el urbanismo paisajístico, han sentado las bases de esta aproximación (Corner, 1997, 1999; Mostafavi \& Doherty, 2010; Reed \& Lister, 2014; Waldheim, 2006).

En esta misma línea de pensamiento se sitúa la construcción de paisajes generativos basados en los ciclos, procesos y flujos que engendran diferentes formas y formatos de interacción con la naturaleza. Se trata de paisajes que también son fuentes y recursos de nuevas sinergias de la ciudad con su medio y que se manifiestan a través de herramientas de infiltración, transporte de sedimentos, fertilización de suelos, protección frente a temporales y otros muchos servicios metabólicos. En la gestión y calibración de esos intercambios es donde el diseño se convierte en director de las capacidades productivas y en configurador de un registro 
legible de complejos y dinámicos paisajes (Fletcher, 2008; Lister, 2007; Orff, 2011; Reed, 2010; Varnelis, 2008).

En este contexto, merece llamar la atención sobre los estudios consistentes en tratar de comprender cuáles son los estados de los ecosistemas que percibimos como estables y útiles para nosotros. De tal modo que como diseñadores deseemos promoverlos e impulsarlos ante una perturbación. Esta perspectiva trasladada al campo del diseño conlleva una aproximación experimental que esté preparada para fallar, en vez de segura ante el fallo. Es decir, aquella que se anticipa a los fallos y en los que el papel del diseño es precisamente el de promover a través de los procesos de cambio de los sistemas su adaptabilidad, de modo que los efectos del fallo en el entorno se minimizan. Esta concepción de modo de fallo seguro reconoce que los sistemas vivos son diferentes a los mecánicos y estructurales, particularmente en el sentido de que regularmente experimentan eventos de cambio que mueven el sistema de un estado aparentemente estable a otro (Ahern, 2011). La idea subyacente es que, si el cambio es inevitable, entonces deberíamos facilitar que ocurran cambios a pequeña escala y poder gestionarlos evitando así un fallo catastrófico (Lister, 2007). Por lo tanto, un diseño adaptativo que sea seguro para fallar en una cuenca hidrográfica propensa a las inundaciones, se basaría en un diseño de paisaje que facilite las inundaciones regulares seguras, en lugar de defenderse y correr el riesgo de una catástrofe, en caso de fallo.

Continuando con el ejemplo presentado al inicio de este artículo, tras Katrina, construir la resiliencia de Nueva Orleans ha significado trabajar no solo para reconstruir la ciudad, sino para
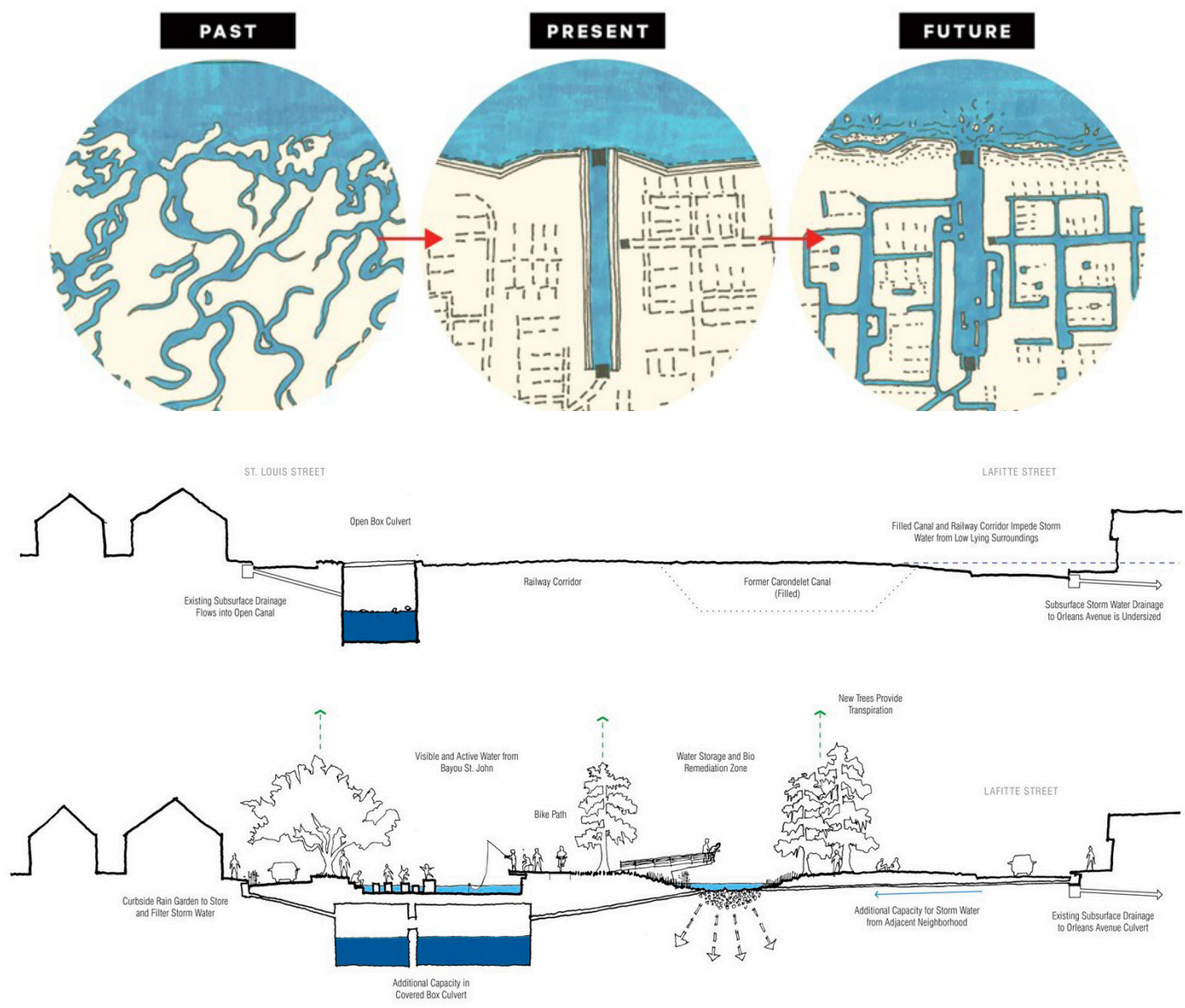

Fuente: Waggoner \& Ball. 
construirla mejor. Una pieza importante de esta reconstrucción pasa por la incorporación de medidas basadas en la naturaleza para gestionar las aguas de escorrentía y minimizar el volumen de aguas a evacuar en caso de fuertes tormentas. Es decir, complementar su infraestructura gris con una infraestructura verde y azul.

El trabajo de planificación del agua de la firma Waggonner. \& Ball para la ciudad de Nueva Orleans se remonta a poco después del huracán Katrina en 2006, con el viaje de David Waggonner a los Países Bajos como parte de una delegación de los Estados Unidos; los talleres Dutch Dialogues y la exposición Living with water, que brindaron una amplia gama de oportunidades para la investigación por diseño y participación de la sociedad. El resultado de todo este trabajo es el Greater New Orleans Urban Water Plan, reconocido como un modelo a seguir por el resto del país y otorgado por la Asociación Americana de Planificación. con el
Premio Nacional de Excelencia en Planificación para la Planificación Ambiental (2015).

El Greater New Orleans Urban Water Plan, supone un cambio en el enfoque de la

región en la gestión del agua, un cambio del riesgo a la resiliencia. Es decir, de luchar contra el agua a convivir con agua. El plan, que incluye tres cuencas hidrológicas que cubren 155 millas cuadradas de terrenos urbanos más 69 millas cuadradas de humedales de retención urbanos, aborda la gestión de aguas pluviales, aguas superficiales y subterráneas.

No se trata solo de perseguir la funcionalidad metabólica, tras esta propuesta de gestión del agua subyace la idea de devolver la ciudad a su condición "pantanosa" original, reintroduciendo el agua y recreando nuevas experiencias urbanas, de mayor calidad, en armonía con su identidad perdida.

Figura 10: Mirabeu Water Garden, uno de los proyectos demostrativos del Urban Water Plan y el espacio público central del futuro "distrito resiliente" de Gentilly.

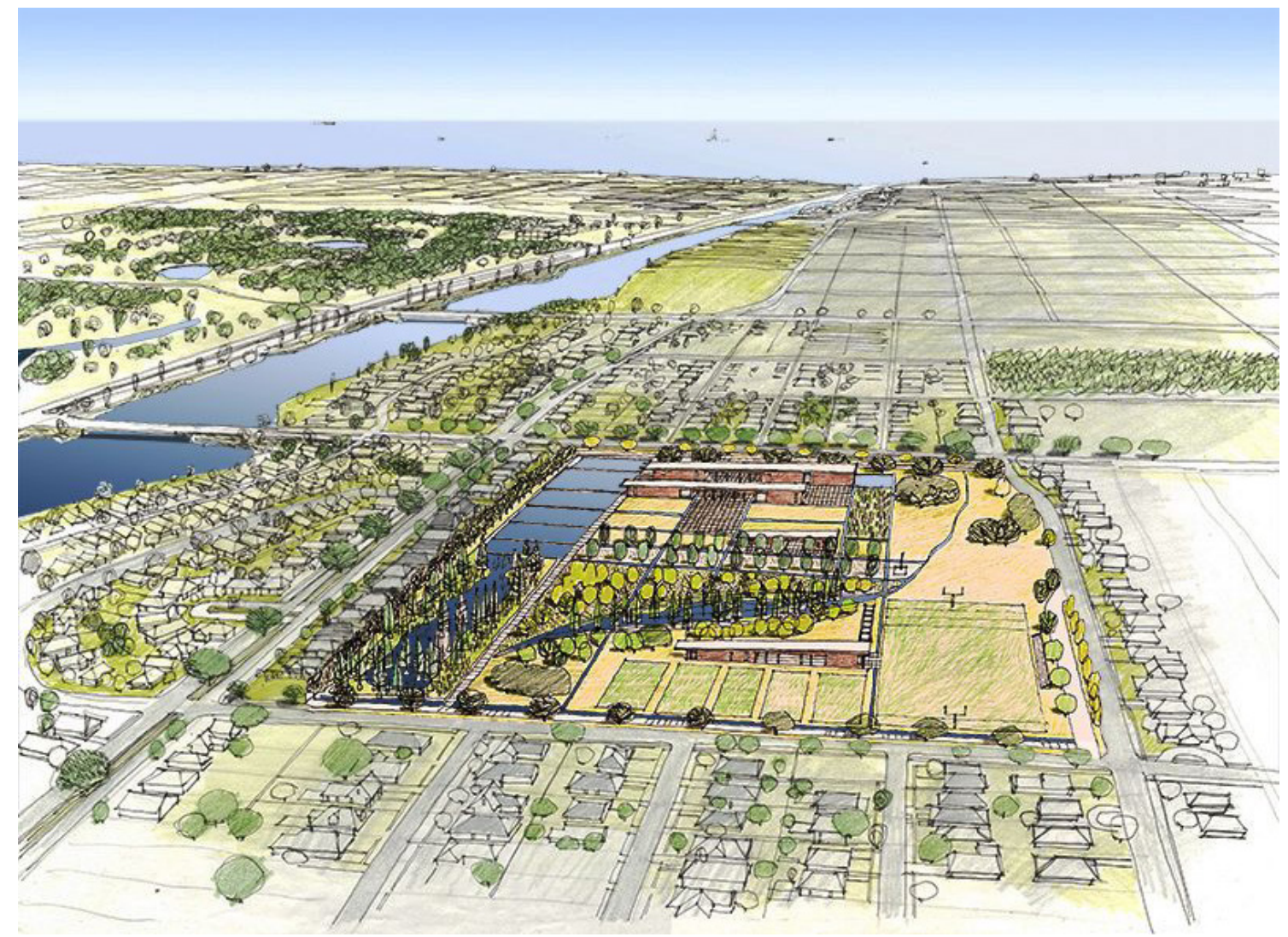

Fuente: Waggonner \& Ball. 


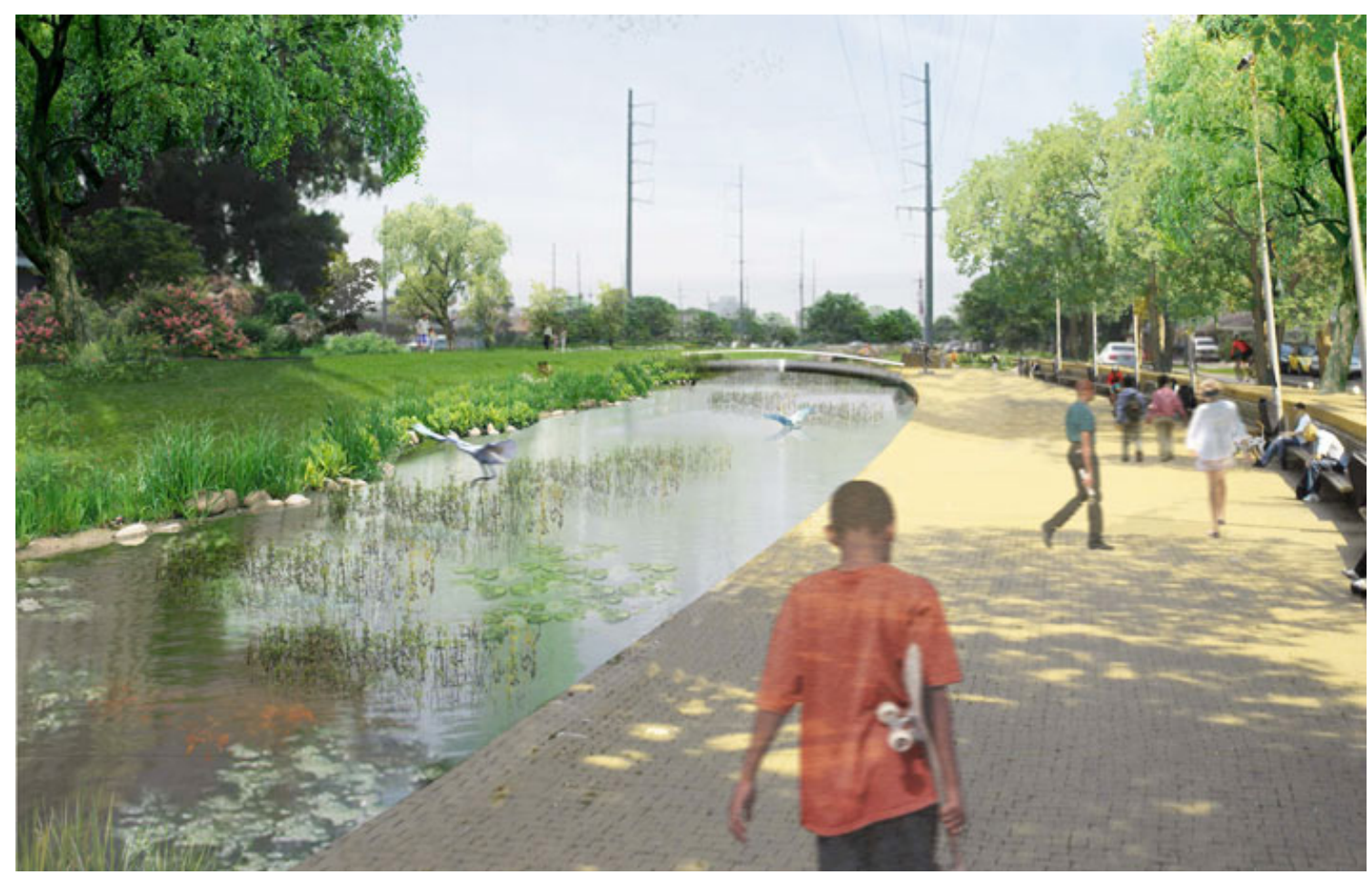

Fuente: Waggonner \& Ball.

El plan y sus proyectos demostrativos, están integrados a escala regional con el Plan de gestión costera de Louisiana, encargado entre otras muchas cuestiones de recuperar los humedales perdidos de la cuenca baja del rio Mis- sissippi, que actúan como protección en caso de temporales. Son proyectos como estos, los que desde la escala del jardín privado hasta la región, articulan la resiliencia de la mano del diseño adaptativo del paisaje.

\section{Bibliografía:}

Allison, H. E., \& Hobbs, R. J. (2004). Resilience, adaptive capacity, and the "lock-in trap" of the Western Australian agricultural region. Ecology and Society, 9 (1).

Ahern, Jack. (2011). From fail-safe to safe-to-fail: Sustainability and resilience in the new urban world. Landscape and Urban Planning, (100), 341-343.

Beck, Ulrich. (1999). World risk society. Cambridge: Polity Press.

Beck, Ulrich. (2015). Emancipatory catastrophism: What does it mean to climate change and risk society? Current Sociology, 63 (1), 75-88.

Berkes, F., Colding, J., \& Folke, C. (2003). Navigating social-ecological systems: building resilience for complexity and change. Cambridge: Cambridge University Press.

Carpenter, S. R., \& Brock, W. (2008). Adaptive capacity and traps. Ecology and Society, 13 (2). 
Clements, Frederic. E. (1916). An Analysis of the Development of Vegetation. Plant Succession. Washington: Carnegie Institution of Washington.

Corner, James. (1997)."Ecology and landscape as agents of creativity”. Thompson, George F., Steiner, Frederick R. Ecological design and planning New York: John Wiley \& Sons.

Corner, James. (1999). Recovering landscape: essays in contemporary landscape architecture. New York, NY: Princeton Architectural Press.

Dewey, John. (1929). The quest for certainty: a study of the relation of knowledge and action. London: George Allen \& Unwin Ltd.

Evans, Geoffrey. R. (2008). Transformation from "Carbon Valley" to a "Post-Carbon Society" in a climate change hot spot: The Coalfields of the Hunter Valley, New South Wales, Australia. Ecology and Society, 13 (1) .

Fletcher, David. Los Angeles River Watershed: Flood Control Freakology. Varnelis, Kazys, The infrastructural city: networked ecologies in Los Angeles. Barcelona: Actar, 2008.

Folke, Carl, 2006: Resilience: the emergence of a perspective for social-ecological systems analysis. Global Environmental Change 16 (3), 253-267.

Folke, Carl., Carpenter, S. R., Walker, B., Scheffer, M., Chapin, T., \& Rockström, J. (2010). Resilience thinking: Integrating resilience, adaptability and transformability. Ecology and Society, 15 (4).

Hay, Colin. (1996). Narrating crisis: the discursive construction of "the winter of discontent." Sociology, 30 (2), 253-277.

Holling, Crawford Stanley. (1973). Resilience and Stability of Ecological Systems. Annual Review of Ecology and Systematics, 4, 1-23.

Holling, Crawford Stanley. (1996). Engineering Resilience versus Ecological Resilience. Engineering Within Ecological Constraints, National Academy of Engineering, Washington, D.C: National Academy Press, 31-44.

Holling, Crawford Stanley. y Gunderson, Lance. (2002): "Resilience and Adaptative Cycles" en Gunderson, Lance y C.S. Holling (Eds.): Panarchy: understanding transformations in human and natural systems (25- 62), EE. UU.: Island Press.

Holling, Crawford Stanley, Gunderson, Lance y Peterson, Garry, 2002: Sustainability and Panarchies. Gunderson, Lance y C.S. Holling (Eds.): Panarchy: understanding transformations in human and natural systems (63-102), EE. UU.: Island Press.

Lance, H., Gunderson, L. H., \& Holling, C. S. (2002). Panarchy. Uderstanding Transformations. Human and Natural Systems (Vol. 49). Washington D.C.: Island Press.

Lister, Nina Marie., (2007). Sustainable large parks: ecological design or designer ecology?. Hargreaves, G., Czerniak, J. (Eds.), Large Parks. Architectural Press, New York: Princeton, NJ, 35-54.

Morton, Timothy. (2013). Hyperobjects: philosophy and ecology after the end of the world. Minneapolis, London: University of Minnesota Press.

Mostafavi, Mohsen., \& Doherty, Garet.(2010). Ecological Urbanism. Cambridge, MA: Lars Müller publishers.

Olsson, P., Folke, C., \& Berkes, F. (2004). Adaptive co-management for building resilience in social- ecological systems. Environmental Management, 34(1), 75-90.

Orff, Kate. Cosmopolitan Ecologies. (2011). Brash, Alexander, Hand, Jamie, Orff, Kate. Gateway: visions for an urban national park. New York, NY: Princeton Architectural Press.

Reed, Chris. The Agency of Ecology. (2010). Mostafavi, Mohsen., \& Doherty, Garet (Eds.), Ecological Urbanism. Cambridge, MA: Lars Müller Publishers.

Reed, Chris, \& Lister, Nina-Marie. (2014). Projective ecologies. Barcelona: Harvard Graduate School of Design and ACTAR.

Schumpeter, Joshep. A. (1942). Capitalismo, socialismo y democracia. London: Routledge.

Turner, Monica. G. (1989). Landscape Ecology: The Effect of Pattern on Process. Annual Review of Ecology and Systematics, 20 (1), 171-197.

Varnelis, Kazys. (2008). Introduction: Networked Ecologies. The infrastructural city: networked ecologies in Los Angeles. Barcelona: Actar. 
Waldheim, Charles. (2006). Landscape as Urbanism. Waldheim, Charles (Ed.), The Landscape Urbanism Reader. New York: Princeton Architectural Press.

Walker, B., Holling, C. S., Carpenter, S. R., \& Kinzig, A. (2004). Resilience, Adaptability and Transformability in Social ecological Systems. Ecology and Society, 9 ,(2), 5.

Walker, Salt, D. 2006. Resilience Thinking: Sustaining Ecosystems and People in Changing World. Washington DC: Island Press.

Westley, \& Frances. (2008). Renewal and Resilience: the role of social innovation in building institutional resilience. In African Health Sciences (Vol. 8, p. 47). Makerere University Medical School. 